\title{
Two-photon exchange in elastic and inelastic electron-proton scattering
}

\author{
S. Kondratyuk*, P. G. Blunden*, W. Melnitchouk ${ }^{\dagger}$ and J. A. Tjon ${ }^{\dagger}$ \\ ${ }^{*}$ Department of Physics and Astronomy, University of Manitoba, Winnipeg, MB, Canada R3T 2N2 \\ ${ }^{\dagger}$ Jefferson Lab, 12000 Jefferson Avenue, Newport News, VA 23606, USA
}

\begin{abstract}
We present an explicit quantum field theoretical calculation of two-photon exchange contribution to elastic electron-proton scattering and to $\Delta$ resonance production in electron-proton collisions.
\end{abstract}

Keywords: Electron-proton scattering, Two-photon exchange, $\Delta$ resonance

PACS: 25.30.Bf, 13.40.Gp, 12.20.Ds, 14.20.Gk

\section{TWO-PHOTON EXCHANGE IN ELASTIC ELECTRON-PROTON SCATTERING}

This work is motivated in part by the apparent disagreement between the Rosenbluth [1] and polarization transfer [2] methods of measuring the nucleon form factors in elastic electron-proton scattering.

The differential cross section for electron-proton scattering can be written as

$$
d \sigma=d \sigma_{B}(1+\delta)
$$

where $d \sigma_{B}$ denotes the usual one-photon exchange (Born) cross section and $\delta$ is the two-photon exchange correction. As two independent kinematical variables one chooses $Q^{2}$, the square of the transferred momentum, and $\varepsilon$, the longitudinal polarization of the photon exchanged in the Born approximation (at fixed $Q^{2}, \varepsilon \rightarrow 0$ and $\varepsilon \rightarrow 1$ correspond to the backward and forward scattering angles, respectively). We determine $\delta$ by explicitly calculating box and crossed-box two-photon exchange loop diagrams [3]. The latter include the nucleon and the $\Delta$ resonance as intermediate hadronic states. The convergence of the loop integrals is ensured by supplying each hadron-photon vertex with a dipole form factor which parametrize the internal structure of the hadrons. We checked that our main results do not depend on the details of this regularizing form factor. It is essential that our calculation treats the nucleon and the $\Delta$ on the same footing since their two-photon exchange contributions have opposite signs.

The effect of the two-photon exchange on the $\varepsilon$-dependence of the differential cross section is demonstrated in Figure 1. These results show that the two-photon exchange contribution, which is traditionally omitted in analyses of the data, can resolve the bulk of the discrepancy between the Rosenbluth and polarization-transfer measurements of the nucleon electromagnetic form factors.

CP842, Particles and Nuclei,

Seventeenth International Conference on Particles and Nuclei

edited by P. D. Barnes, M. D. Cooper, R. A. Eisenstein, H. van Hecke, and G. J. Stephenson

(C) 2006 American Institute of Physics 0-7354-0338-4/06/\$23.00 


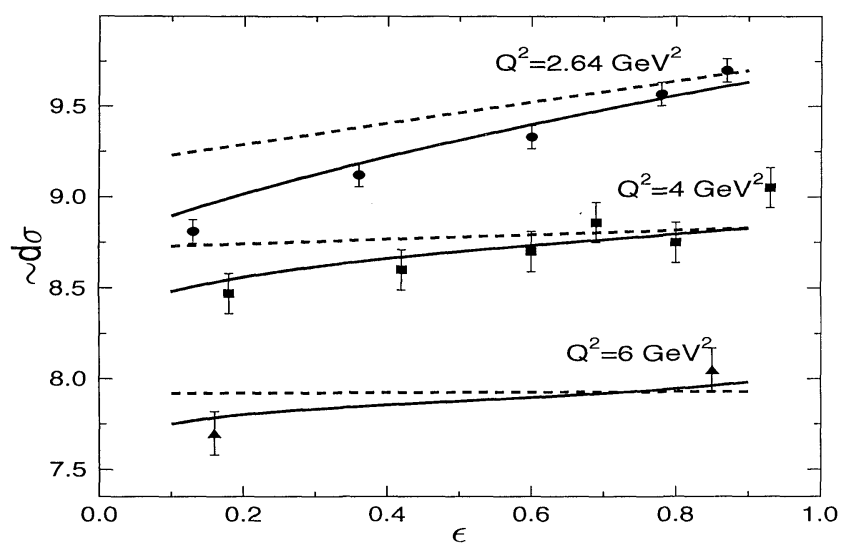

FIGURE 1. Effect of the two-photon exchange contribution on the (scaled) cross section for elastic electron-proton scattering, corresponding to various $Q^{2}$ values. The data points are from the Rosenbluth measurements [1]. The dashed lines show the Born cross section alone, using the form factors measured in the polarization-transfer experiments [2]. The solid curves include the additional two-photon exchange contribution. The dipole form factor cutoff $\Lambda=0.84 \mathrm{GeV}$ was used in all hadron-photon vertices entering the loop diagrams with the intermediate nucleon and $\Delta$ states.

\section{TWO-PHOTON EXCHANGE EFFECTS FOR THE $\Delta$ PRODUCTION REACTION}

The two-photon exchange effects are also quite important in the production of the $\Delta$ resonance in electron-proton collisions. For this process, in addition to the box and crossed-box exchange diagrams with nucleon and $\Delta$ intermediate states, we included a loop diagram with a contact $\gamma \gamma N N$ vertex which is necessary for the calculation to satisfy gauge invariance. The $\varepsilon$-dependence of the two-photon correction to the $\Delta$ production cross section is shown in Figure 2. It is noteworthy that by studying the nonlinearity of the two-photon exchange contribution for the $\Delta$ production process [4] one may be able to obtain additional information on the $\gamma \Delta \Delta$ coupling constant, a scarcely known but much needed ingredient in nuclear physics (such as studies of isobar contributions to the deuteron wave function).

The present calculation is fully relativistic and obeys the properties of crossing symmetry and gauge invariance. These are important model-independent constraints, especially at low energies and momentum transfers.

In conclusion, we have shown that the theoretical analysis of the data, for both elastic and inelastic electron-proton scattering, must include two-photon exchange effects in addition to the traditionally considered Born contribution.

\section{ACKNOWLEDGMENTS}

We thank John Arrington and Vladis Tvaskis for useful discussions. 


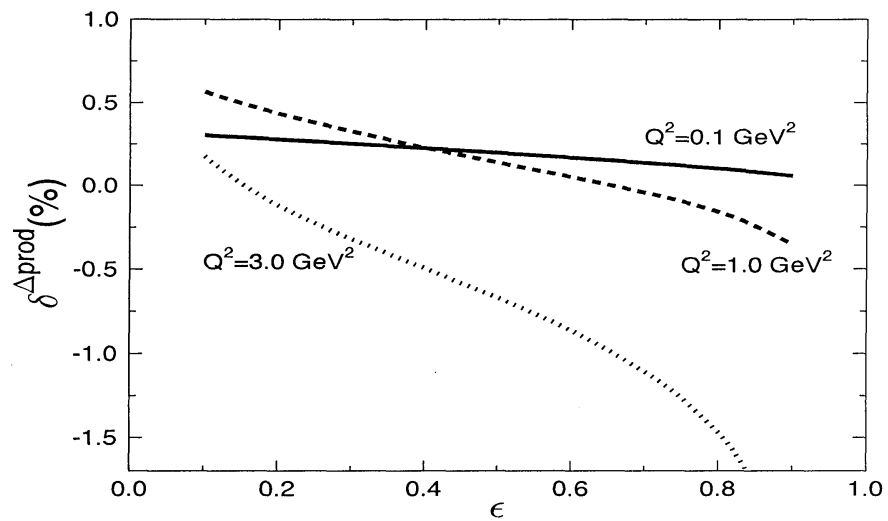

FIGURE 2. Two-photon exchange contribution to the cross section for $\Delta$ production in electron-proton scattering. The dipole form factor cutoff $\Lambda=0.84 \mathrm{GeV}$ was used in all hadron-photon vertices entering the loop diagrams with the intermediate nucleon and $\Delta$ states. The curves are labeled by the values of the momentum transfer squared.

\section{REFERENCES}

1. L. Andivahis et al., Phys. Rev. D 50, 5491 (1994); I. A. Qattan et al., Phys. Rev. Lett. 94, 142301 (2005).

2. O. Gayou et al., Phys. Rev. Lett. 88, 092301 (2002).

3. S. Kondratyuk, P. G. Blunden, W. Melnitchouk, and J. A. Tjon, Phys. Rev. Lett. 95, 172503 (2005);

P. G. Blunden, W. Melnitchouk, and J. A. Tjon, Phys. Rev. C 72, 034612 (2005).

4. V. Traskis et al., nucl-ex/0511021. 